\section{INSTITUCIONES PÚBLICAS Y DINÁMICA EMPRESARIAL EN EL SECTOR CULTURAL: LA ARQUEOLOGÍA COMERCIAL EN ESPAÑA}

\author{
Eva Parga-Dans \\ Instituto de Ciencias del Patrimonio (INCIPIT), CSIC
}

Manuel Fernández Esquinas

Instituto de Estudios Sociales Avanzados (IESA), CSIC.

\section{PUBLIC INSTITUTIONS AND FIRM DYNAMICS IN CULTURAL INDUSTRIES: THE COMMERCIAL ARCHAEOLOGY IN SPAIN}

\begin{abstract}
The cultural industries are gaining importance due to its social and economic role in knowledge society. In countries with a rich and ancient history such as Spain it is especially relevant the conservation and management of heritage, not only because its cultural importance, but also because a new business activity has emerged close to it: the sector of commercial archaeology. However, there is an important gap in the understanding of these cultural and heritage services. It is difficult to observe the conditions that shape its emergence, the economic and labour situation of the firms and the opportunities for development of these firms. This article presents a study of Spanish commercial archaeology, a knowledge intensive business service which has grown in close relation with heritage regulations and policies since 1990's. We explore the emerging process and the current conditions of this sector by using several empirical sources: regulations and policy documents, a census of archaeological companies, and a survey to these companies. The case study illustrates the dynamics created by the public sector and its influence on the creation of a special market, as well as the influences on innovation and economic growth of cultural industries.
\end{abstract}

KEY WORDS: Economic sociology; innovation; institutional innovation; contract archaeology; knowledge intensive business services; archaeological sector; heritage sector.

\section{INTRODUCCIÓN}

Las transformaciones sociales y económicas derivadas del advenimiento de la llamada sociedad del conocimiento han generado un cambio profundo en las estructuras productivas de los países desarrollados. Si bien la producción industrial de manufacturas había sido el motor de desarrollo durante el siglo XIX y buena parte del siglo XX, en la actualidad la oferta/demanda de servicios se constituye como actividad relevante de las sociedades de capitalismo

\begin{abstract}
RESUMEN: El sector cultural y las actividades que comprende están cobrando relevancia debido a su impacto socioeconómico en las sociedades del conocimiento. En paises con una rica y dilatada historia cultural como España, destaca especialmente la conservación y gestión del patrimonio, que ha dado lugar al surgimiento del mercado de la arqueología comercial. No obstante, existe una notable dificultad para observar la situación de los servicios culturales y patrimoniales, de las condiciones que influyen en su surgimiento, su situación económica y laboral y sus posibilidades de desarrollo. Este artículo realiza un análisis de la arqueología comercial española, un servicio intensivo en conocimiento que surge y se desarrolla en las políticas y regulaciones de patrimonio histórico surgidas desde la década de 1990. Utilizando fuentes empíricas documentales, un censo de las empresas de arqueología, y una encuesta a una muestra de empresas, se analizan las condiciones y el proceso que da lugar al surgimiento de este sector. Este caso de estudio ejemplifica las dinámicas desarrolladas por la administración pública y su influencia en la creación de un mercado, así como en el ritmo y dirección de la actividad económica y la innovación en el sector cultural.
\end{abstract}

PALABRAS CLAVE: Sociología económica; innovación; innovación institucional; arqueología comercial; servicios intensivos en conocimiento; sector arqueológico; sector patrimonial.

avanzado. Más aún, cierto tipo de servicios se consideran fundamentales para la dinamización económica y social de las regiones. No se trata sólo de que en las sociedades modernas el empleo y la actividad económica de los servicios sea ya predominante, sino de que la composición y orientación de los servicios tienen un efecto en la innovación y en el desarrollo económico en general. Existe un consenso creciente sobre la especial relevancia que adquieren el conocimiento y los servicios basados en el conocimiento (Ettlie y Rosenthal, 2011). Entre ellos, los servicios cultura- 
les se revelan como un conjunto de actividades prósperas y dinámicas que son capaces de generar renta y empleo (Vicente, 2007). Por esta razón cada vez es más habitual la proliferación de trabajos de investigación en torno a lo que se ha denominado Economía de la Cultura (Greffe, 1990). Por ejemplo, en este sentido lo establece la Comisión Europea en la Agenda de Lisboa (2000): se estima que este tipo de actividades implicaron un 2,4\% del empleo total en el año 2005 y que, además, el tipo de empleo que promueve este sector es cualificado (un 48\% posee titulación universitaria), a pesar de que se caracteriza por su elevada temporalidad (16\%) (Eurostat, 2007).

Estos datos permiten intuir el importante papel que está desempeñando el sector cultural. No obstante, un problema habitual es que las actividades y servicios culturales y patrimoniales no suelen desagregarse en las encuestas sectoriales $y$, por tanto, difícilmente son identificables en las clasificaciones económicas. Tampoco se han desarrollado estadísticas especializadas o indicadores en torno a esta temática, y los estudios sobre empresas culturales suelen centrarse sobre todo en los campos artístico y audiovisual. La amplitud en la definición de la cultura (y la abundancia de definiciones sobre esta actividad) refleja la necesidad de homogeneizar parámetros y comenzar a sentar las bases de estudio en torno a estas actividades. En términos socioeconómicos se entiende la cultura como el sector que incluye al patrimonio artístico y monumental, archivos, bibliotecas, libros y prensa, artes visuales, arquitectura, artes escénicas y audiovisuales multimedia; entendidos como ámbitos que desarrollan funciones de conservación, creación, producción, comercio y formación (Eurostat, 2007). Esta situación ha creado la necesidad de definir y desarrollar una mejor comprensión de los vínculos entre conocimiento, servicios, cultura, desarrollo social y económico. Se trata por tanto de un campo en el que queda mucho trabajo por desarrollar para lograr percibir empíricamente el impacto socioeconómico de las actividades culturales, donde es necesario comenzar haciendo estudios focalizados en sectores específicos como el de la arqueología.

Este trabajo realiza una aproximación al sector cultural y patrimonial a través de las actividades económicas asociadas a la arqueología. Para ello, se parte de la línea de investigación en Socioeconomía del Patrimonio iniciada en el Laboratorio de Patrimonio (IEGPS-CSIC), que tiene por objeto recopilar información y analizar los efectos y beneficios en términos sociales y económicos del patrimonio, como bien (material e inmaterial) y como sector y servicio. En este artículo se avanza el estado de la investigación hasta el momento y los principales resultados referidos al sector empresarial.

Los objetivos del trabajo son los siguientes. En primer lugar, se pretende realizar un análisis descriptivo del sector arqueológico comercial en nuestro pais, haciendo hincapié en los factores que conducen a esta nueva demanda de servicios. En segundo lugar, se trata de caracterizar a las empresas de arqueología españolas en varias de sus dimensiones relevantes: cómo son estas empresas, qué ofrecen, cuál es su perfil y su mercado de trabajo. En tercer lugar, se pretende observar cómo son los procesos de innovación en este sector. Se trata sobre todo de indagar los elementos de dinamización tecnológica y productiva vinculados a esta actividad, y explorar aquellos factores que contribuyen a su sostenimiento económico.

Además de la caracterización estructural y de mercado de esta actividad comercial, este trabajo realiza una reflexión sobre los factores institucionales que promueven la emergencia de un sector económico. De hecho, la estructuración de la arqueología comercial y la profesionalización de esta actividad es resultado de la normalización legal de la gestión patrimonial y de la creación de un aparato administrativo dedicado a desempeñar esta labor, lo que ha generado una necesidad en torno a este tipo de servicios. Por ello, el sector público toma especial relevancia como elemento dinamizador del entorno industrial. En este contexto, la arqueología comercial constituye un servicio creativo, basado en el conocimiento, altamente cualificado y que se configura como un recurso con implicaciones en el ámbito económico y social, pero con importantes problemas estructurales. En definitiva, es un buen ejemplo para comprender las implicaciones de la gestión pública en el mercado y en los ritmos y direcciones de los procesos de innovación en el resto de los servicios.

La base empírica sobre la que se basa el trabajo parte de un intenso proceso de recogida y sistematización de información sobre la labor administrativa y reguladora patrimonial. Del mismo modo, dado que no existen fuentes de datos en este contexto, se ha realizado una búsqueda exhaustiva de las empresas que participan en el sector y una encuesta 
en la que se recoge información sobre sus características y su dinámica productiva. El artículo consta de los siguientes puntos. Después de esta introducción, se describe el contexto sociopolítico y los procesos de transformación institucional en gestión de patrimonio arqueológico desarrollados en España (apartado 2). A continuación se presenta un modelo de análisis dirigido a caracterizar este sector en términos de la innovación que genera (apartado 3). Seguidamente, se explica la metodología seguida en el estudio (apartado 4). Los resultados se presentan a través de una descripción de los factores institucionales que condicionan el sector (apartado 5), de la caracterización de la oferta de servicios arqueológicos reflejados en el perfil de las empresas (apartado 6) y de la situación de las empresas en el contexto de crisis económica, así como de sus actividades de innovación (apartado 7). Finalmente, el artículo se cierra con una serie de conclusiones e implicaciones para el desarrollo empresarial del sector cultural (apartado 8).

\section{Marco SOCIOpolitico y CAMbio institucional EN LA GESTIÓN DEL PATRIMONIO ARQUEOLÓGICO}

Las transformaciones institucionales tienen consecuencias en la composición económica y en la dirección de los mercados, y no sólo en los modelos de organización social y en las estructuras de los estados. En este sentido, el sector cultural/patrimonial es un claro exponente de su dependencia del contexto político y normativo. La oferta de servicios arqueológicos se configura en España como una nueva actividad comercial estructurada en relación a una serie de hitos institucionales que tienen lugar en el último cuarto del siglo XX.

En la década de los sesenta comienza un significativo crecimiento de las ciudades. Inicialmente los procesos de construcción se llevaban a cabo sin control urbanístico y con escasa consideración de los bienes con valor histórico o cultural, situación que provocó la destrucción de una gran cantidad de patrimonio. Para impedir los efectos nocivos de este proceso, y siguiendo las directrices europeas, a principios de los ochenta se empieza a sentir la necesidad de regular la situación. La actuación principal es la aprobación de la Ley de Patrimonio Histórico Español en 1985, con el objeto de proteger, preservar y gestionar el patrimonio del país. A partir de este momento se han ido desarrollando una serie de requisitos para la protección y gestión de patrimonio, que han generado la necesidad de contar con profesionales en gestión de patrimonio arqueológico para la realización de este tipo de actividades. Esto a su vez condujo a la formación de pequeñas empresas, que fueron adquiriendo experiencia en la materia, y que a la vez fueron diversificando su experiencia y creando valor, dando lugar a un nuevo "nicho" de mercado habitualmente denominado arqueología comercial (también arqueología profesional o contractual). Esta consiste en la compra y venta, u oferta y demanda, de una serie de servicios vinculados a la gestión del patrimonio arqueológico (Parga-Dans, 2011).

A raíz de estos cambios surge también en España la gestión patrimonial. Este término se refiere al conjunto de actividades destinadas a la protección y difusión del patrimonio cultural (Querol, 2010), lo cual se concreta en una serie de servicios variados: de documentación (conocimiento, catalogación e inventariado de bienes patrimoniales para su protección), de intervención (evaluación de impacto en el patrimonio ante intervenciones en el terreno), de puesta en valor (valorización patrimonial mediante su conversión en bienes o recursos culturales), de consultoría (asesoría especializada sobre temas que afecten al ámbito patrimonial) y de difusión (acercamiento a la sociedad de conocimientos y recursos patrimoniales) (Parga-Dans, 2011).

Previamente al proceso descrito, la actividad arqueológica se ceñía al ámbito académico. Con la normalización legislativa en materia patrimonial y con la estructuración de un aparato gestor se establecen una serie de requisitos en materia de protección patrimonial que las administraciones no pudieron satisfacer debido a los recursos limitados con los que contaban. Esta actividad comienza a ser demandada a profesionales externos a la administración, habitualmente profesores e investigadores en el ámbito académico, y luego progresivamente comienzan a demandarse profesionales formados en patrimonio, tanto en el ámbito de la administración como en el de la empresa. En este último caso, comienzan a demandarse servicios especializados en gestión de patrimonio, lo que da lugar al surgimiento de nuevas empresas que canalizan las salidas laborales de los nuevos titulados, que en ciertos casos están potenciadas por profesionales que provienen del mundo universitario. Otro factor importante en la constitución de este nuevo

ARBOR Vol. 188753 enero-febrero [2012] 135-152 ISSN: 0210-1963

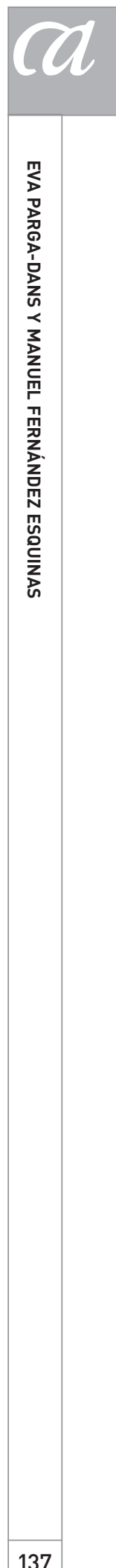


nicho de mercado es el proceso de subcontratación realizado con los profesionales autónomos y las nuevas empresas especializadas, realizado por la propia administración o por las empresas que actúan en ámbitos sensibles al patrimonio arqueológico, donde tienen una papel relevante las empresas constructoras. La normativa provoca que en la gran obra pública, y también en las construcciones en general realizadas en terrenos con protección patrimonial, se demanden estudios de prospección y valorización, que normalmente no son asumidos por las empresas del sector, sino que se contratan a empresas especializadas que reúnen profesionales y conocimientos adecuados. Esto da lugar a la puesta en valor de un amplio patrimonio que es necesario indagar antes de que la actuación sobre el terreno tenga consecuencias irreversibles sobre el posible patrimonio, lo que a su vez provoca la expansión de la demanda de servicios en todas las facetas de la gestión patrimonial citadas antes.

Este modelo tiene paralelismos con otros surgidos en paises occidentales. El nacimiento de la vertiente comercial de la arqueología en el contexto español sigue un proceso similar al desarrollado en el ámbito norteamericano y británico, donde esta actividad surge como resultado de la normalización legal de la protección del patrimonio (National Historic Preservation Act, en el caso de Estados Unidos en 1966, y Ancient Monument and Archaeological Areas Act, en Reino Unido en 1979). Estos modelos, que implican una gestión "parcialmente privada" del patrimonio arqueológico, convergen en unos contextos en los que se desarrolla una importante actividad constructiva y en los que el neoliberalismo emerge como modelo imperante (siendo impulsores relevantes las políticas de Margaret Tatcher en Inglaterra a partir de 1979 y Ronald Reagan en Estados Unidos a partir de 1980).

No obstante, en el modelo español hemos de añadir otro hito institucional que tiene lugar entre 1979 y 1983, y que condiciona de manera importante la estructura de este tipo de servicios: el proceso de transferencia de competencias en materia de patrimonio del gobierno central a las comunidades autónomas, lo cual derivará en el modelo actual de gestión de patrimonio arqueológico. En este período las comunidades autónomas comenzaron a desarrollar sus propios modelos de gestión y sus reglamentos destinados al control de las actividades arqueológicas en sus respectivos territorios (Querol y Martínez, 1996). En suma, se observa un proceso a través del cual el sector público establece un marco estructural que tiene consecuencias en el entorno productivo, en este caso creando las condiciones, las infraestructuras y los modelos de gestión que generan una nueva oferta de servicios y así un mercado de trabajo. Este proceso es entendido en este trabajo como aquellos factores institucionales que enmarcan la actividad económica, y que condicionan los procesos productivos que se llevan a cabo en el sector. Se trata de un proceso en el cual la formalización de un nuevo nicho de mercado se desarrolla a partir de las transformaciones institucionales y tecnológicas. Esto significa que la gestión del patrimonio arqueológico deja de estar relegado al ámbito de la investigación, para convertirse en servicio y conformar un nuevo sector de actividad que tiene un impacto social y económico.

\section{Hacia un marco de anÁlisis para el estudio de la actividAd en el SECtor aroueológico}

A continuación se exponen las bases conceptuales que sirven de herramientas analíticas para estudiar el sector arqueológico español de carácter comercial, tanto en sus aspectos socioeconómicos como en el especial papel que desempeña el entorno institucional público como agente dinamizador de este mercado. Para ello se recurre a diferentes aproximaciones teóricas que resultan especialmente apropiadas para el sector debido a que dan cuenta de algunos de sus rasgos distintivos. A saber, la perspectiva de los sistemas de innovación (Freeman, 1987; Lundvall, 1992; Nelson y Winter, 1982; Edquist, 2005; Malerba, 1992; Teece y Pisano, 1994; Dosi, Marengo y Fagiolo, 1998; Metcalfe, 1998), algunos enfoques de la actual sociología económica (Granovetter, 1985; Smelser y Swedberg, 2005), la corriente que se ocupa de los servicios intensivos en conocimiento (Miles, 1995; Windrum y Thomlinson, 1998; Thomi y Böhn, 2003; Vence y González, 2009), y algunos estudios que consideran el papel del Estado en la generación de sectores económicos.

En cuanto al enfoque de los sistemas de innovación, se trata de una aproximación teórica que considera los procesos de innovación como factores determinantes de la creación del bienestar y riqueza de las sociedades, como un elemen- 

las empresas, lo cual ha generado un interés renovado en el análisis de sus factores y consecuencias desde la vertiente empresarial, científica y política. El aspecto central es que las prácticas innovadoras no se desarrollan únicamente en el ámbito de la empresa, sino de manera sistémica (Lundvall, 1992) y vinculada con el entorno inmediato en sus varias vertientes académica, institucional y productiva. Este modelo tiene una concepción interactiva de los información, donde el conocimiento es el aspecto clave para el desarrollo de las competencias empresariales; lo cual está íntimamente relacionado con la capacitación del capital humano y su habilidad para generar nuevo conocimiento (que adquiere relevancia frente al desarrollo tecnológico), que a su vez puede ser aplicado en nuevos productos y servicios (Foray, 2004). Por lo tanto, el análisis de la innovación desde esta aproximación está íntimamente vinculado a las transformaciones institucionales, organizativas $y$, en definitiva, sociales. Esto implica un aprendizaje y cambio de gestión de recursos y activos, interacciones entre agentes, capacidades para enfrentarse a nuevos problemas y la búsqueda de herramientas para combinar en la resolución de los mismos, lo cual tiene consecuencias para las posibilidades de que surja un modelo económico creativo y transformador. No obstante, es necesario resaltar que uno de sus problemas inmediatos es que la complejidad del proceso innovador es difícilmente cuantificable.

Una serie de estudios convergentes con los anteriores son los enfoques empresariales realizados desde la sociología económica. Desde esta perspectiva, la empresa, como agente del entorno productivo, es analizada en una doble dimensión, en tanto que es parte del sistema económico y del sistema social. Por una parte, es una organización que forma parte de un sistema económico, sancionado por el mercado que coordina la actividad productiva para contribuir a la generación de riqueza. Por otra parte, es también parte de un sistema social que engendra formas de cooperación, reglas de comportamiento, valores, lenguajes, símbolos y procesos de socialización. En este contexto, la dimensión institucional, tanto la formal referida a las reglas, como la informal reflejada en los marcos cognitivos y normativos, se considera como elemento fundamental en el desarrollo y desempeño económico de las empresas (Smelser y Swedberg, 2005). to fundamental para el crecimiento y la competitividad de procesos de innovación, prestando atención a los flujos de
En tercer lugar, desde el lado de los estudios dirigidos al uso del conocimiento, son de resaltar los que interpretan las relaciones entre los distintos agentes como estructuradas a partir de la acumulación de conocimiento, donde igualmente cobra especial relevancia el rol desempeñado por las instituciones. Las empresas son habitualmente entendidas como agentes que deben soportar el cambio técnico para adaptarse a cualquier incentivo económico (Dosi, Orsenigo y Labini, 2005). En economías globalizadas, uno de los factores que mejora la acumulación de las empresas es contar con una serie de elementos que les permite aumentar la capacidad de absorción de conocimiento y transformarlo de manera adaptada a los procesos productivos. Normalmente, la presencia de servicios y actividades intensivas en conocimiento, externos o internos a la empresa, es lo que facilita la capacidad de absorción. Por tanto, la generación de conocimiento se entiende como una dinámica sistémica de intercambio socialmente distribuido, dependiente de instituciones como elementos primordiales, y como un importante factor que acelera el proceso de creación de capacidades (Foray, 2004).

Finalmente, es importante tener en cuenta los efectos de las políticas públicas que, a menudo, influyen en la dirección y ritmo de los procesos de innovación. A saber, el entorno legislativo puede influir en las decisiones de abrir o cerrar negocios, en la consideración de qué mercados pueden ser más beneficiosos para invertir o cuáles se deberian evitar, en qué estructuras y prácticas de empleo deben adoptar las empresas, y en cómo éstas deben competir (Dosi, Orsenigo y Labini, 2005). El sector público juega un papel central en la creación, mantenimiento y desarrollo del entorno industrial, como agente que demanda y/o gestiona servicios y/o productos, marcando los ritmos y direcciones de los procesos de innovación (Gregersen, 1992).

Un objetivo prioritario del sector público suele ser el de crear un entorno industrial dinámico en el que los mercados puedan desarrollarse, por lo que para ello desde el sector público se implementan diferentes tipos de mecanismos tales como políticas, leyes, niveles de inversión, fijación de estándares, soporte financiero, infraestructuras, I+D, formación, subsidios, tasas, subvenciones, etc. A través de los modelos legislativos y de gestión se define la dirección y el ritmo de la innovación, se impulsa o se inhibe el crecimiento económico, se establecen los niveles de calidad y el tamaño de los mercados (Gregersen, 1992).

ARBOR Vol. 188753 enero-febrero [2012] 135-152 ISSN: 0210-1963

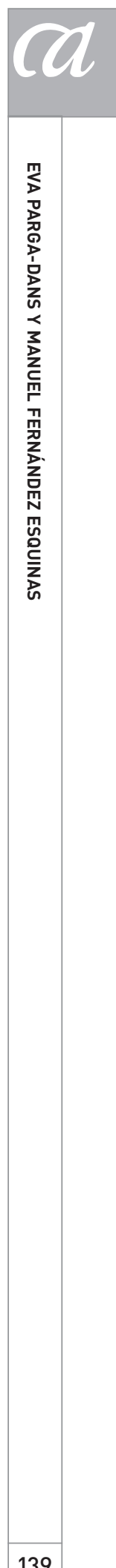

doi: 10.3989/arbor.2012.753n1009 
Los reglamentos desarrollados por las administraciones pueden ser reactivos o proactivos en su carácter. Según B. Gregersen (1992), los modelos reactivos, también denominados "sticks", hacen referencia a políticas basadas en "castigos" y ejercen el cumplimiento de la norma a través de sistemas de impuestos, multas, tarifas y cuotas. Por otro lado, estarian los modelos proactivos, también denominados "carrots" (Gregersen, 1992,145), que identifican políticas basadas en "premios". Estos inducen al comportamiento a través de sistemas de subsidios, contratos de desarrollo, fomento del aprendizaje, subvenciones directas, I+D, infraestructuras, apoyo financiero, etc. De este modo, los diferentes modelos normativos influirán en el diseño de los mercados y en los procesos de innovación que se den en ellos.

Los anteriores fundamentos definen los pilares para la construcción de un enfoque analítico dirigido a comprender cómo son los procesos de surgimiento y desarrollo de empresas, así como de sus procesos productivos, en el sector arqueológico español. Se trata de un mercado que emerge a través de una transformación institucional. El análisis del entorno institucional es, por tanto, una dimensión especialmente relevante para comprender la caracterización de esta actividad así como la naturaleza de la innovación desarrollada en un sector. La hipótesis de partida de este trabajo se articula, por lo tanto, a partir de la vinculación existente entre el entorno institucional y el entorno productivo y en los efectos que esta relación produce en las dinámicas predominantes en los sectores empresariales afectados. A saber, en el caso de actividades generadas por una regulación estatal, surgen nichos de mercado que generan empresas que crean valor y empleo y dan lugar a dinámicas creativas. En sus inicios este sector es normalmente frágil debido a que se encuentra en una situación subordinada, que lo hace depender de una serie reducida de clientes, cuya demanda viene condicionada por los imperativos que marca la regulación. Esto le hace vulnerable a las coyunturas de la actuación pública, sobre todo de las crisis económicas. Ahora bien, las posibilidades de desarrollo de las empresas de este sector dependerán de las dinámicas y capacidades que adquieran las empresas. En concreto, serán las empresas más innovadoras las que tengan mayores posibilidades de mantenerse en el mercado de manera menos dependiente de las coyunturas. El caso de estudio del sector arqueológico español reúne las claves para analizar los efectos que el proceso de estructuración de la política patrimonial tiene en la emergencia de esta oferta de servicios, así como la situación y la capacidad de respuesta de las empresas.

\section{Metodología}

Para estudiar el sector comercial de la arqueología existe una notable dificultad debida a la inexistencia de fuentes oficiales o secundarias que sistematicen la información y la actividad generada en torno a este mercado. Por ello, en este trabajo se han desarrollado tareas dirigidas a describir y analizar la arqueología comercial española, el entorno político en el que se desarrolla y los elementos que inciden en el desarrollo tecnológico y productivo de este sector, lo cuál tiene un componente descriptivo que resulta necesario para empezar a analizar un colectivo sobre el que existe escasa información empírica. En concreto, se han realizado las siguientes tareas: construir un mapa de identificación y localización de agentes/organizaciones del entorno institucional e industrial del sector arqueológico español; crear un registro sobre la oferta de actividad existente; y diseñar una estrategia de observación e investigación a través de encuesta que posibilite la recopilación de datos relevante de las empresas del sector.

\subsection{Análisis del entorno institucional}

En primer lugar se ha llevado a cabo un proceso de recogida de datos por comunidades autónomas con el objeto de recopilar información sobre el sector público, la normativa y los agentes con responsabilidades en materia de patrimonio. Inicialmente se identificaron los departamentos de la administración competentes en materia patrimonial y las personas encargadas de los mismos. Posteriormente se contactó con todos ellos para recopilar información sobre la actividad del sector arqueológico por comunidad autónoma, el perfil y tamaño de estos departamentos, y la normativa patrimonial vigente a través de entrevistas semi-dirigidas. Del mismo modo, se han empleado los registros sobre licencias de excavación arqueológica como indicador de la evolución del sector. En esta actividad participaron ocho de las diecisiete comunidades autónomas. A pesar de que no se recoge el conjunto del territorio español, la información abarca situaciones que permiten 
analizar los rasgos institucionales que afectan a las empresas en varios contextos.

\subsection{Registro de la población operativa de empresas}

En segundo lugar se ha construido una base de datos centrada en el ámbito de la oferta. Debido a las dificultades para localizar a los agentes que ofertan servicios arqueológicos (sobre todo, la ausencia de CNAE propio) se ha tomado como unidad básica de análisis la empresa arqueológica, dejando al margen otros agentes que también ofertan estos servicios, como los trabajadores por cuenta propia (autónomos) y por cuenta ajena, para los que no se encontró ningún registro, y que además constituyen un colectivo muy variable. Actualmente la base de datos consta de 273 casos de empresas de arqueología localizadas que se encuentran activas en el año 2009. Estas empresas se han registrado acudiendo a diferentes fuentes (es de resaltar que no existe ningún registro oficial, homogéneo y actualizado):

- datos facilitados por las administraciones autonómicas con competencias en patrimonio,

- colegios profesionales de licenciados en filosofía y letras con departamentos de arqueología,

- bases de datos de empresas a través del código nacional de actividad económica (como el Sistema de Análisis de Balances Ibéricos),

- anuncios publicitarios,

- a través del empleo de la técnica de muestreo denominada "bola de nieve", en la que diferentes informantes o participantes en el estudio refieren otros casos que son incluidos (Frey, Botan, Kreps, 2000, 133).

Es necesario tener en cuenta que los registros de la base de datos no tienen por qué reflejar la totalidad de empresas de arqueología localizadas en España, ni el total de agentes que ofertan servicios arqueológicos en nuestro país. No obstante, constituye la primera sistematización de datos en torno a este mercado en el contexto español. En todo caso, se trata de una población de carácter estratégico que comprende una parte relevante del sector en España (al menos la formada por empresas con cierta entidad), y que por lo tanto resulta adecuada para explorar este sector y tratar de contrastar los supuestos de partida del estudio.

\subsection{Diseño y características de la encuesta}

A partir de la fuente anterior se ha recopilado información socioeconómica a través de una encuesta. El diseño del cuestionario dirigido a empresas se orientó principalmente a aspectos de carácter socioeconómico. Para la estructura y organización del cuestionario se ha tomado como modelo de referencia la Encuesta sobre Innovación Tecnológica en las Empresas, desarrollada por la Institución Nacional de Estadística, e inspirada en el Community Innovation Survey (CIS) de la Unión Europea. Posteriormente se adaptaron las preguntas y se introdujeron otras más específicas sobre el sector. El desarrollo de esta parte específica del cuestionario estuvo apoyado por un panel de expertos en gestión de patrimonio. Finalmente, el cuestionario quedó estructurado en torno a los siguientes bloques temáticos: Características generales de la empresa, Actividad económica, Opiniones, actitudes y comportamientos, Actividad innovadora, Formación y difusión, Relaciones con otros agentes del sector, Impacto de la crisis en el sector.

Teniendo en cuenta la dispersión geográfica de las empresas en el territorio español y la pretensión de recopilar información para todo el universo poblacional, se diseñó una estrategia de recogida de datos secuencial a través de una aplicación on-line para que las empresas pudiesen cumplimentar el cuestionario de manera autoadministrada. Este proceso se completó con la utilización del sistema informático interactivo Computer Assisted Telephone Interviewing (CATI), lo que ha permitido que entrevistadores formados para esta tarea hayan aumentado la cantidad de respuestas obtenidas y hayan mejorado la calidad de los datos cumplimentando cuestionarios inacabados mediante la realización de sucesivas llamadas telefónicas.

Esta estrategia ha permitido obtener una tasa de respuesta del 77\%. De las 273 empresas que constituyen la base de datos, 212 participaron en el estudio. La elevada aceptación que ha tenido esta iniciativa ha generado un importante volumen de datos con un sesgo reducido de respuesta y un bajo nivel de error. Se puede considerar, por tanto, que la muestra representa con cierta fidelidad la situación del sector. Finalmente se codificó y procesó la información utilizando el programa estadístico Statistical Package for the Social Sciences (SPSS 14,0) y se elaboró un mapa de frecuencias globales y por comunidad autónoma a partir del cual se realizó el análisis de datos que sirve de base a este trabajo.

ARBOR Vol. 188753 enero-febrero [2012] 135-152 ISSN: 0210-1963 


\section{LOS CONDICIONANTES DE LA ACTIVIDAD aRQUeOLÓgICA: SITUACIÓN EN LAS COMUNIDADES Autónomas}

Este apartado se dedica a exponer las principales características del contexto en el que surgen las empresas de arqueología, lo cual tiene que ver estrechamente con el entorno institucional y entorno productivo existente en las comunidades autónomas en las que se ubican las empresas. Para ello se utiliza como fuente principal de datos el registro de empresas en estos territorios y las fuentes documentales y orales obtenidas de los gobiernos autonómicos.

\subsection{El proceso de constitución de empresas de arqueología}

La arqueología comercial española nace y se desarrolla tras la publicación de la Ley de Patrimonio Histórico de 1985. Por esta razón, la fecha de constitución de las empresas que actualmente forman parte de la base de datos $(n=212)$ representa una variable fundamental para establecer la relación con la actividad política y reguladora.

Con anterioridad a la publicación de esta normativa tan sólo dos empresas del total de casos analizados estaban trabajando, en este caso en la Comunidad de Madrid, lo que refleja la práctica inexistencia de este mercado. Es a partir de los años noventa cuando esta actividad comercial comienza a desarrollarse en todo el territorio español, comenzando en aquellas comunidades que fueron pioneras en el desarrollo de la normativa autonómica en materia de protección patrimonial (Castilla-La Mancha 1990, Andalucía 1991, Cataluña 1993, Galicia 1995, Aragón 1999, Madrid 1998, etc.). Durante esta década se constituye el $27 \%$ de las empresas de arqueología encuestadas. El mayor desarrollo de empresas tiene lugar durante el período 2000-2005: en este período emerge el 40\% de las empresas que forman nuestra fuente. Cabe señalar que en estos años casi todas las Comunidades Autónomas han publicado ya sus normativas en materia de protección patrimonial. Por lo tanto, se observa a través de este gráfico cómo este mercado se desarrolla paralelamente al proceso normativo.

Además, este gráfico refleja la coyuntura económica del contexto español en esta época y la evolución que han seguido estas empresas. Durante el período 2000-2005 surgen la mayoría de las empresas que forman la oferta de servicios arqueológicos, un período coincidente con la bonanza económica del sector de la construcción en España, que ha sido uno de los motores del desarrollo económico de nuestro pais, y también el principal demandante de los servicios de arqueología. Durante el período 20052009 desciende drásticamente la creación de empresas de arqueología, siendo éste un efecto de la crisis económica que afecta gravemente al sector a partir del año 20062007, debido a la paralización de la demanda vinculada con la construcción y al proceso de recesión económica cuyos efectos aún se padecen. En suma, observando el proceso de creación de empresas se constata que el sector público, a través de su actividad normativa y gestora, ha desempeñado un importante papel en la dinamización de este mercado, aunque recientemente es la coyuntura económica, en un contexto de crisis, la que paraliza el crecimiento del sector.

\subsection{El volumen de actividad arqueológica}

Lo indicado anteriormente se constata más claramente si analizamos los datos de volumen de actividad arqueológica a través del número de actuaciones concedidas por las administraciones autonómicas. Dado que se trata de un sector parcialmente intervenido, para la realización de muchas de las actividades de gestión arqueológica es necesario contar con autorización administrativa. Esta es la fuente de datos que permite analizar la evolución de la actividad (véase Gráfico 2).

En términos generales se observa una tendencia de aumento de autorizaciones durante el período analizado, desde el año 2001 hasta el año 2006, lo que se corresponde con la tendencia del proceso de constitución de empresas. La actividad arqueológica registra un período de crecimiento sostenido desde el año 2001 que se ve paralizado en el año 2006 en algunos casos, y a partir del año 2007 de manera generalizada. Este decrecimiento de la actividad arqueológica es más acusado en las regiones en las que el sector de la construcción y, por tanto, el arqueológico había sido más próspero.

Cabe señalar que se observan diferentes volúmenes de actividad entre las Comunidades Autónomas: Cataluña y Comunidad Valenciana presentan los valores más eleva- 
GRÁFICO 1. FECHA DE CONSTITUCIÓN DE EMPRESAS DE ARQUEOLOGÍA ESPAÑOLAS EN RELACIÓN A LA COMUNIDAD AUTÓNOMA
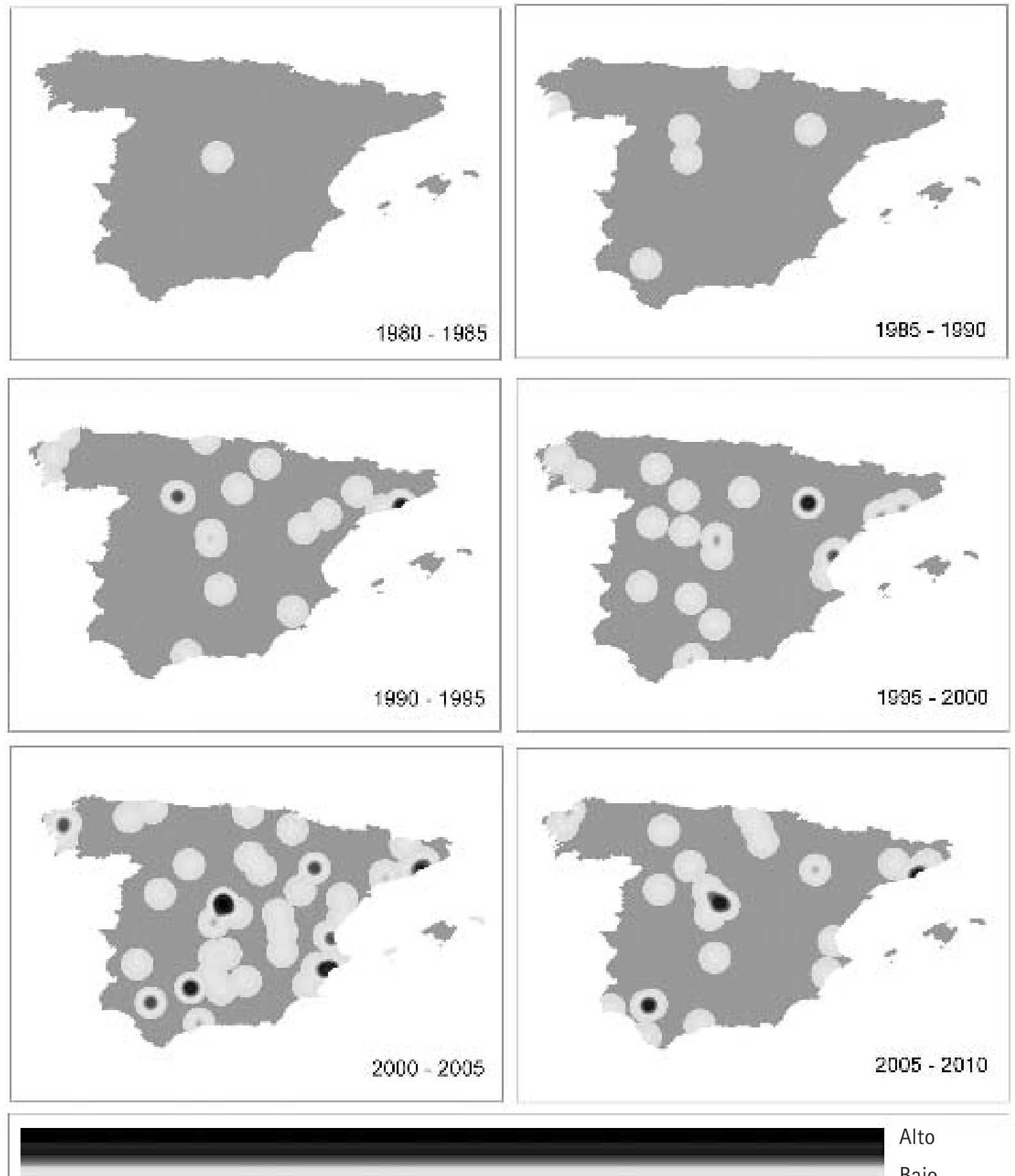

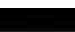
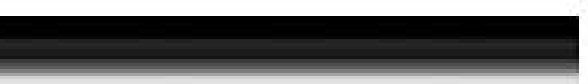

Alto

Bajo

Fuente: Parga-Dans, 2011. 
GRÁFICO 2. DATOS DE VOLUMEN DE ACTIVIDAD ARQUEOLÓGICA POR COMUNIDADES AUTÓNOMAS. PERMISOS CONCEDIDOS POR LA ADMINISTRACIÓN

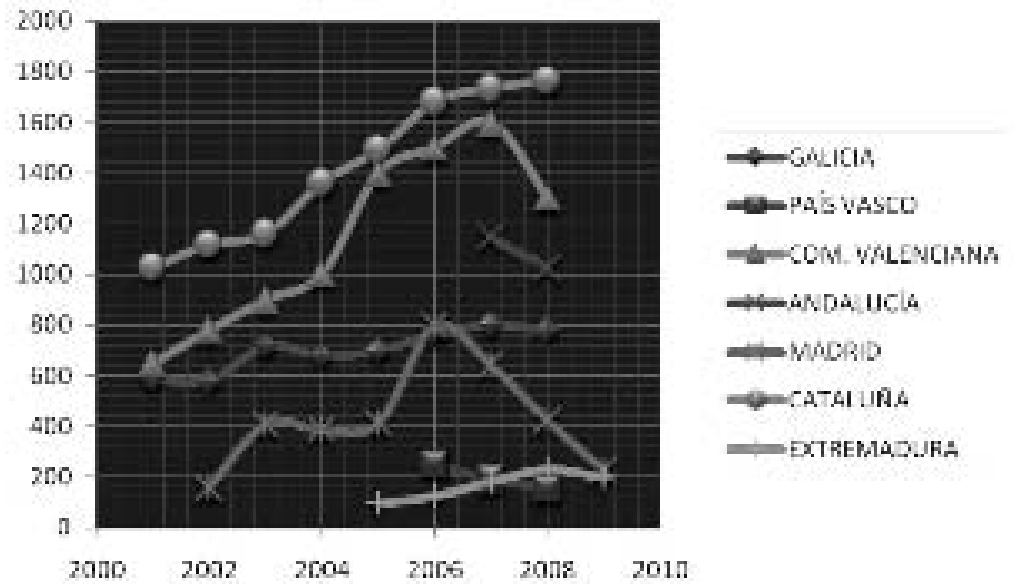

Fuente: Parga-Dans, 2011

dos, siendo Extremadura y País Vasco las que registran el número más bajo de autorizaciones. A pesar de que las dinámicas por Comunidades Autónomas son diferentes, se observa un cambio de tendencia a partir del año 2007 en la Comunidad Valenciana, Andalucía, Extremadura y Galicia. Tras un período de intenso crecimiento en el caso de la Comunidad Valenciana, y tras un período de moderado crecimiento en Galicia, el volumen de la actividad arqueológica empieza a decrecer en 2007. Esta caída empieza antes en Madrid, en 2006, y en el País Vasco se observa la misma situación. Este hecho puede explicarse a través de la estrecha vinculación de la actividad arqueológica con el sector de la construcción, que ha sido muy importante para el desarrollo de la oferta de servicios de arqueología. Estas empresas han venido desarrollando una importante actividad vinculada con el sector constructivo a través de los servicios de evaluación y de prevención de impacto en patrimonio. Son tareas previas al proyecto de construcción, que implican una metodología arqueológica exigida por una empresa (en la construcción o el sector de la ingeniería), el gobierno o un cliente privado, cuando su trabajo tiene un impacto en el territorio que podría dañar el patrimonio cultural protegido.

Por ello, la actual crisis económica está teniendo un fuerte efecto sobre la arqueología comercial, especialmente en las comunidades que tienen un gran sector de la construcción y que habian experimentado un repunte en la actividad arqueológica, como es el caso de Cataluña, Comunidad Valenciana, Andalucía y Madrid. Sin embargo, en regiones como Galicia y el País Vasco el sector arqueológico ha crecido de manera moderada, y como resultado los efectos de la crisis son menos acentuados. De nuevo se observa una estrecha relación entre el entorno institucional y el productivo a través de los permisos otorgados por las administraciones para el desarrollo de actuaciones arqueológicas, aunque no toda la actividad desarrollada por estas empresas tiene que ser supervisada por la administración. A continuación se analiza el número de empresas por Comunidades Autónomas y se indican algunas claves sobre los diferentes modelos de gestión.

\subsection{Localización de las empresas y modelos de gestión}

En este apartado caracterizamos los factores de localización de las empresas de arqueología y nos preguntamos si la mayor o menor concentración de empresas en un área determinada está vinculada a los modelos de gestión distintivos de las Comunidades Autónomas. Se observa que el mayor número de empresas se sitúa en las Comunidades de Andalucía, Cataluña, Madrid (con más de 30 organi- 


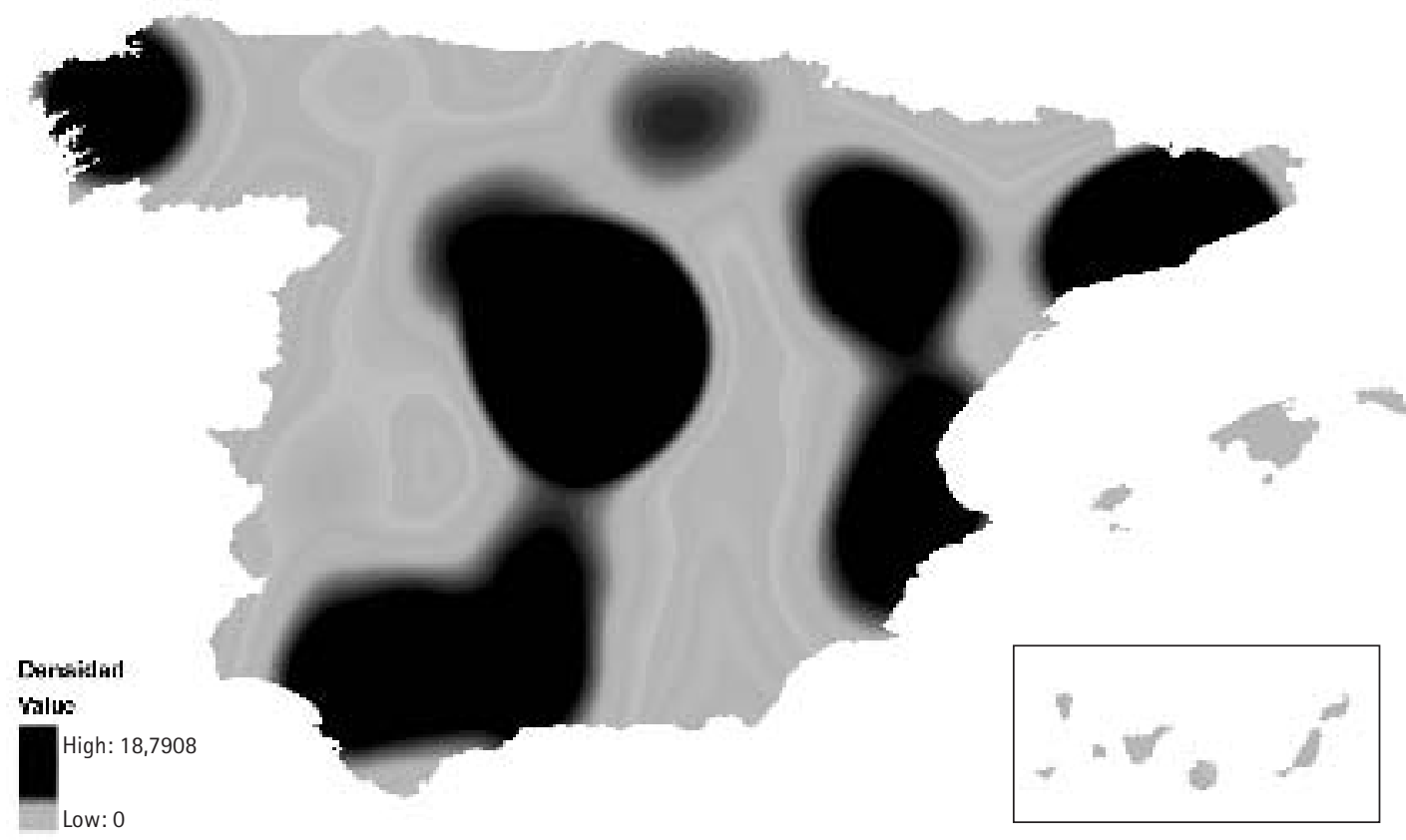

zaciones). Concretamente, existen elevados patrones de concentración en las ciudades de Madrid y Barcelona, así como en los términos municipales que rodean estas áreas. Con menor densidad destacan las zonas en torno a SeviIla, Córdoba y Málaga. Las Comunidades Autónomas que registran más de 20 casos son Castilla y León, Comunidad Valenciana, Galicia y Aragón, seguidamente Castilla-La Mancha (con más de 10 empresas), mientras que el resto de Comunidades Autónomas tienen menos de 10 empresas. Otras ciudades que registran una concentración significativa son Zaragoza, Valencia y Santiago de Compostela.

De acuerdo con la importancia que tiene la localización geográfica para los mercados, cabe preguntarse si los modelos de gestión patrimonial y legal desarrollados en estos territorios han determinado el mayor tamaño de este mercado en las Comunidades de Andalucía, Madrid y Cataluña. A continuación se realiza una breve descripción, teniendo en cuenta la situación más significativa de estas regiones, utilizando para ello la información documental y las en- trevistas realizadas a responsables de gestión patrimonial en los departamentos autonómicos correspondientes. Del conjunto de Comunidades Autónomas, se han escogido tres ejemplos que consideramos reflejan las pautas más relevantes.

En la Comunidad de Andalucía la gestión del patrimonio arqueológico se desarrolla desde la Dirección General de Bienes Culturales, donde estaban trabajando ocho arqueólogos en los servicios centrales durante el año 2009. La protección del patrimonio se lleva a cabo a través de la Ley $1 / 1991$, de Patrimonio Histórico de Andalucía, modificada por la posterior Ley 14/2007. Además, durante este mismo año se aprueba un reglamento específico sobre la actividad arqueológica (Decreto 168/2007). El proceso normativo llevado a cabo en esta comunidad autónoma parece indicar un procedimiento amplio, detallado y continuado en el tiempo. Actualmente están trabajando en este contexto cuarenta y cinco empresas de arqueología, lo que supone un importante tamaño de este sector en una región

ARBOR Vol. 188753 enero-febrero [2012] 135-152 ISSN: 0210-1963 
caracterizada por un importante volumen de patrimonio arqueológico y un importante tamaño en el contexto español (en términos de superficie, población y PIB).

Otra comunidad que registra un importante desarrollo de actividad arqueológica es Madrid, que cuenta con treinta y ocho empresas y un importante mercado de trabajo para la arqueología (se estima que están trabajando en esta comunidad más de seiscientos arqueólogos). Sin embargo, la normativa de protección patrimonial es muy general, no se publica hasta el año 1998 (Ley 10/1998, de Patrimonio Histórico de Madrid) y no se publica ningún decreto que especifique los criterios para su protección. En cambio, en esta comunidad se desarrolla un importante movimiento asociacionista en relación a la profesión arqueológica a través de dos organizaciones: la Asociación Madrileña de Trabajadores y Trabajadoras de Arqueología (AMTTA) y la Sección de arqueología del Colegio de Doctores y Licenciados en Filosofía y Letras de Madrid. A pesar de que el número de actuaciones arqueológicas en esta comunidad no es el más elevado del territorio, las organizaciones empresariales establecidas habitualmente trabajan para otras regiones. Madrid, como capital y centro administrativo del país, es la región en la que se establecen las grandes sedes administrativas, las grandes organizaciones empresariales y, por tanto, también se localiza uno de los más importantes volúmenes de profesionales de arqueología. Se observa una importante actividad comercial, frente a un aparato gestor y legislativo menos desarrollado y menos intervencionista, El desarrollo de la actividad arqueológica en este territorio parece más bien fruto de la localización estratégica de la demanda de estos servicios, sobre todo las grandes empresas y los entes del Gobierno del Estado.

Finalmente, el contexto catalán se caracteriza por disponer de un desarrollado aparato gestor y legal para la protección del patrimonio arqueológico. Esta región tiene un servicio específico dedicado a la arqueología y paleontología en el que trabajan veintidós personas, en colaboración con siete arqueólogos territoriales. La actividad arqueológica está regulada por la Ley 9/1993, de Patrimonio Cultural de Cataluña, y por el Decreto 78/2003, sobre protección del Patrimonio Arqueológico. En Cataluña se ha registrado un importante crecimiento de la actividad patrimonial, cuenta con importantes infraestructuras en materia de gestión y localiza uno de los mercados más importantes para la arqueología comercial en España (44 empresas), además las empresas de mayor tamaño. También existe una amplia trayectoria asociacionista a través de la Asociación de Arqueólogos de Cataluña y un Convenio Colectivo de Trabajo para el Sector de la Arqueología y la Paleontología en Cataluña (2008). Ésta fue la primera comunidad en conseguir aprobar un convenio de estas caracteristicas y única hasta el año 2009, momento en que también se aprobó un convenio en Galicia.

A través de esta breve descripción se observa que el desarrollo de la arqueología comercial en estas comunidades parece responder a diferentes patrones, aunque el denominador común sea la estructuración de este sector como resultado de la normalización legal en estos territorios. EI caso andaluz registra un voluminoso mercado de trabajo debido al tamaño de esta comunidad y al patrimonio arqueológico que posee. En el caso de Madrid el desarrollo del mercado parece responder a la localización estratégica de la demanda de servicios arqueológicos en el centro neurálgico del país. Lo mismo sucede en el caso catalán, que además ha implementado un desarrollado modelo regulador. Es de resaltar que en todos los casos presentados la arqueología comercial ha tenido una evolución creciente desde su nacimiento. Esto permite concluir que no parecen existir elementos determinantes en los modelos de gestión autonómicos que hayan marcado diferencias territoriales en lo referido a la limitación de la actividad productiva, o por lo menos los datos registrados no lo demuestran. La información analizada muestra diferentes estructuras administrativas territoriales (en tamaño, presupuestos, normativas, volumen de actividad, etc.) en áreas en las que este sector se ha desarrollado de manera creciente.

\section{Perfil de las empresas dedicadas a la aroueología}

En este apartado se expone una sintesis de la información recogida en la encuesta. Se ha realizado una selección de los datos que permite caracterizar la oferta de servicios haciendo hincapié en las debilidades y en las oportunidades, como elementos sobre los que es necesario establecer estrategias para promover la dinamización tecnológica y productiva de este sector. 
Una de las principales caracteristicas de estas empresas, al igual que del mercado de trabajo generado en torno a la arqueología comercial (y que es analizado a través del procedimiento de creación de empresas) es la frágil estructuración de este sector. El sector público es el entorno que crea las condiciones que favorecen la generación de esta oferta de servicios, cuyo desarrollo y evolución parece estar más vinculado a la coyuntura económica que a la estructura institucional. A través de los resultados de la encuesta se han contabilizado 2.358 personas que estaban trabajando en las empresas de arqueología españolas a finales del año 2008. De ellas, un total de 457 personas son empresarios y/o socios, 573 son empleados indefinidos y 1.328 personas tienen contratos temporales. A pesar de que estos datos hay que considerarlos como una estimación de una parte del mercado de trabajo vinculado a la arqueología (empresas identificadas), es de apreciar la elevada temporalidad en los puestos de trabajo, aspecto recurrente en el sector cultural (Eurostat, 2007).

En términos generales, las empresas de arqueología se caracterizan por su reducido tamaño: un $47 \%$ de las encuestadas están formadas por dos socios y un $27 \%$ por sólo uno. Además, el $37 \%$ de las mismas no tiene ningún empleado indefinido y un $25 \%$ tiene tan sólo un empleado.
Por lo tanto, estamos hablando de microempresas ( 2 ó 3 personas) que contratan personal eventual en función del volumen de trabajo. Este reducido tamaño se constata también a través de los datos de facturación: el 30\% de las empresas facturan anualmente entre $10.000 € \mathrm{y}$ $75.000 €$. Se refleja con estos datos que el gran número de empresas que proliferan a partir de la década de los noventa del siglo pasado están marcadas por la fragilidad, al disponer de una facturación anual que las sitúa al borde de la supervivencia, la cual se ve gravemente afectada con la finalización del contexto de bonanza económica de los años noventa. Por otra parte, contrasta con el anterior perfil un grupo de empresas (10\%) que tienen un mayor tamaño: facturan al año más de $500.000 €$ y emplean entre 10 y 31 personas de manera indefinida. Estos casos serían ejemplos de las empresas de arqueología consolidadas en el mercado.

La estrecha dependencia de esta actividad comercial con el sector de la construcción se observa de manera clara si analizamos el tipo de clientes/usuarios de los servicios de las empresas de arqueología: son en mayor medida las empresas de infraestructuras (36\%) y constructoras (21\%), por encima de la demanda pública, que supone alrededor de un $27 \%$.

GRÁFICO 4. PRINCIPALES CLIENTES DE LAS EMPRESAS DE ARQUEOLOGÍA. DATOS EXPRESADOS EN PORCENTAJES

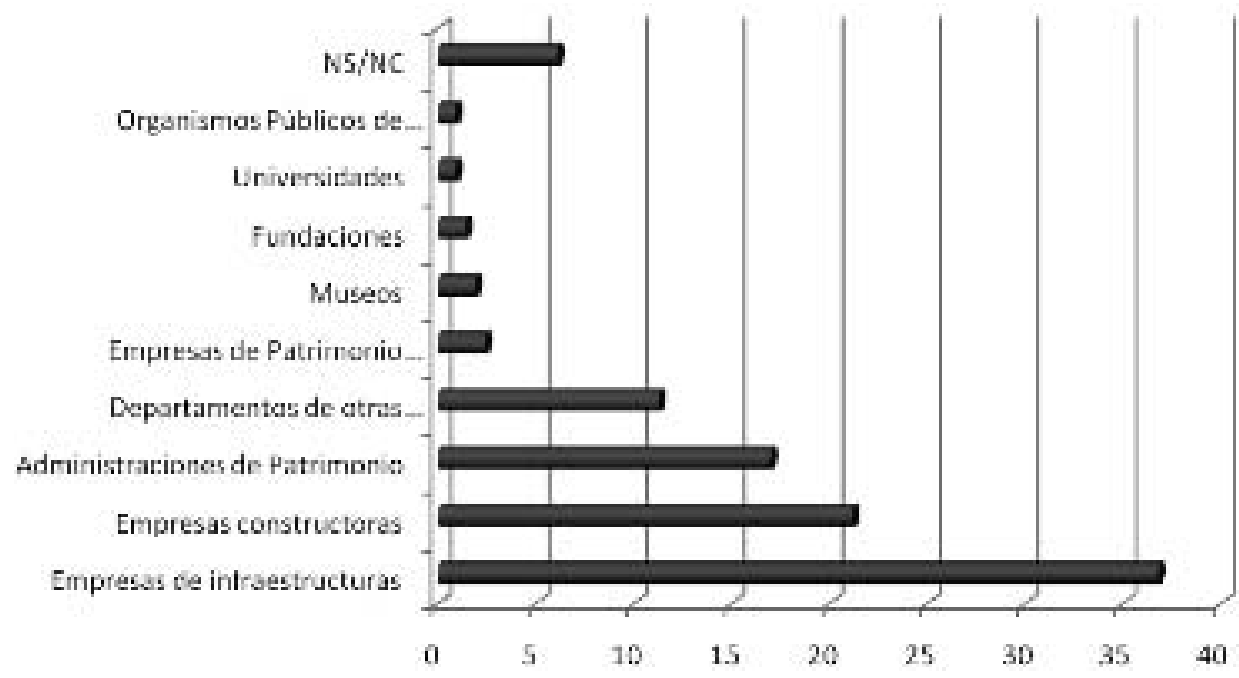

Fuente: Parga-Dans, 2011. 
Esta caracterización de la demanda de servicios arqueológicos resulta relevante debido a que perfila un sector que nace con el objeto de ofrecer servicios en torno a la gestión del patrimonio. Sin embargo, su demanda real de servicios está orientada a la actividad constructiva a través de la evaluación de impacto arqueológico. Esta relación fue sin duda positiva para el crecimiento y sostenimiento del sector durante la etapa de desarrollo de la construcción, aunque ha tenido graves efectos para la consolidación del mercado con el advenimiento de la crisis económica y en ausencia de otras estrategias de fortalecimiento del sector arqueológico. A ello hay que unir los problemas inherentes a la heterogénea regulación de la protección patrimonial de las comunidades autónomas, que supuso que el ámbito de actuación de estas empresas se vinculase al área geográfica más próxima. Se trata por tanto de un mercado segmentado territorialmente en función del desarrollo institucional, por lo que se perfila como un sector emergente débil y con importantes problemas para consolidar su posición, agravado además por el contexto de crisis económica y la ausencia de incentivos institucionales para su desarrollo. Es de resaltar que los incentivos públicos son a través de la regulación, o bien de la contratación directa, aunque las administraciones no han establecido un marco de ayuda que facilita el desarrollo tecnológico y productivo de estas empresas.

Ahora bien, aunque la supervivencia de estas empresas y profesionales en el mercado atraviesa por una complicada situación, existen otro tipo de elementos asociados a la oferta de servicios arqueológicos que suponen una oportunidad para su desarrollo, y que tienen que ver con la elevada cualificación del personal y la caracterización de estos servicios como intensivos en conocimiento. El personal vinculado a este sector destaca por tener un elevado nivel de estudios y amplia experiencia en gestión del patrimonio. Alrededor de un $70 \%$ del personal estable de las empresas de arqueología (socios y contratados indefinidos) tienen estudios universitarios y un 15\% dispone de estudios de tercer ciclo. Esta elevada cualificación es un componente esencial de este sector, que oferta servicios desarrollados a través de un conocimiento técnico especializado, lo que a su vez requiere relaciones directas y personalizadas con los clientes. Este hecho supone que un componente importante en este mercado es la localización estratégica de las empresas, que habitualmente se establecen en aquellos núcleos urbanos en los que se encuentran las principales sedes de las instituciones públicas y de las empresas constructoras, inmobiliarias e ingenierías, que son las principales usuarias y demandantes de los servicios de arqueología (esta es también una de las razones por la que en Madrid, Cataluña y Andalucia se concentran un gran número de empresas).

\section{Caracterización de la innovación en el Sector AROUEOLÓGICO}

Dadas las características y los recursos de que disponen las empresas que prestan servicios intensivos en conocimiento (Miles, 1995; Windrum y Thomlinson, 1998; González, 2007), es posible calificar algunas de ellas como empresas innovadoras (por ejemplo, en la clasificación estadística según la CNAE, el 33\% de los casos se reconoce a través de los códigos 73 y 74, dedicados a la investigación y desarrollo, y a otras actividades empresariales, como servicios técnicos, jurídicos, de consultoría, asesoramiento, etc., lo que refleja un componente intelectual alto (Thomi y Böhn, 2003). La elevada cualificación del personal es lo que les permite a ciertas empresas adaptarse a situaciones cambiantes y sobrevivir en el mercado, lo que en ocasiones supone una importante oportunidad para la dinamización tecnológica y productiva de esta actividad comercial.

Para observar en qué medida las empresas responden a la situación económica es importante analizar los procesos de innovación, aunque no es fácil caracterizar los procesos de innovación en este sector. Según las definiciones oficiales, estos procesos se definen como "la introducción de un nuevo, o significativamente mejorado, producto (bien o servicio), de un proceso, de un nuevo método de comercialización o de un nuevo método organizativo, en las prácticas internas de la empresa, la organización del lugar de trabajo o las relaciones exteriores" (OCDE, 2005, 56). Habitualmente, los elementos para la medición de la innovación están relacionados con la inversión en $\mathrm{I}+\mathrm{D}$, con la generación de patentes o modelos de utilidad o con la realización de una serie de productos nuevos o mejoras en los productos y servicios que se prestan. Estos indicadores son difícilmente cuantificables en el sector servicios y más aún en el sector patrimonial, lo cual oculta los posibles procesos innovadores. 
En este caso es posible sostener a nivel macro que un importante proceso de innovación por sí mismo consiste en el elemento de transformación institucional (la introducción de una norma) y cuya consecuencia o valor es la creación de una actividad comercial para la gestión de un "bien social", el patrimonio. El elemento desencadenante son las administraciones públicas, que provocan una demanda, generando un mercado, y promoviendo la creación de un bien público. Ahora bien, vistos desde el punto de vista de la empresa, es de destacar que los procesos de innovación, en lo referido a las tecnologías propias desarrolladas por las propias empresas, son poco importantes: a menudo se reducen a la compra de equipamiento o software para llevar a cabo sus actividades. Sin embargo, a través del análisis de las actividades se intuye que el carácter de las innovaciones reside sobre todo en la transformación del conocimiento disponible. Se trata de la combinación de un conocimiento teórico, desarrollado habitualmente en el ámbito académico de las humanidades, con vestigios materiales que otras culturas han ido dejando en el territorio, y que o bien era desconocido hasta el momento en el que se realiza la intervención arqueológica, o bien no se consideraba como valioso. La explotación sistemática de materiales y vestigios es lo que conlleva un elemento creador y transformador, que está aportando un valor económico y social. En primer lugar, a través de la generación de un mercado de trabajo. En segundo lugar, en la creación de capacidades para actuar con el patrimonio, donde adquieren enorme importancia factores no tecnológicos difícilmente cuantificables, sobre todo las relaciones directas con los clientes. En tercer lugar, la diversificación de la oferta de servicios en gestión de patrimonio realizada por las empresas, que ofrece la posibilidad de ampliar y hacer sostenible el sector.

En lo referido a la diferenciación de la respuesta de las empresas, se observa que las más adaptativas, estables y dinámicas son aquellas que presentan mayor tamaño, las cuales a su vez registran un mayor volumen de facturación. Estas empresas son igualmente las que invierten en actividades de formación, desarrollan actividades de divulgación y establecen convenios de cooperación con otros agentes. Es de resaltar que, de la muestra recopilada, tan sólo un $10 \%$ de las empresas cumplen estas características, pudiéndose tomar como ejemplo de buenas prácticas para la dinamización del sector. Por lo tanto, parece que para lograr una mayor estabilidad y consolidación en el merca- do es necesario contar con unos recursos mínimos y una masa crítica. Una vez alcanzado este umbral se generan importantes oportunidades productivas. Con una mayor masa crítica es posible acceder a proyectos 0 atender demandas de mayor envergadura, diversificar la oferta de servicios, formalizar acuerdos de cooperación, conseguir

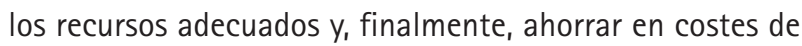
producción y amortizar la inversión realizada. Este "círculo virtuoso" a su vez favorece la generación de conocimiento y otorga mayor capacidad para invertir y desarrollar procesos de innovación, lo cual redunda en cotas de estabilidad y disminuye el riesgo de sucumbir a las coyunturas e incertidumbres del mercado.

\section{Conclusiones}

En este artículo se ha analizado el proceso de emergencia y desarrollo de la arqueología comercial española como una oferta de servicios que tiene lugar en el contexto institucional específico en el que esta actividad está imbricada. La arqueología comercial comienza a desarrollarse en España a partir de la entrada en vigor de la legislación en materia de patrimonio arqueológico y de la puesta en marcha de las infraestructuras de gestión a nivel nacional y autonómico. Este proceso ejemplifica el importante papel desempeñado por el entorno institucional y por el sector público a través del establecimiento de las condiciones, infraestructuras y modelos de gestión que dinamizan los mercados. En el contexto arqueológico, la publicación de la Ley de Patrimonio Histórico Español (1985) crea un marco institucional que gestiona el cambio técnico a través de la puesta en marcha de un nuevo "nicho" de actividad. Esta transformación desarrollada desde el sector público y con importantes consecuencias en el mercado es entendida como un proceso de innovación institucional.

Además, esta actividad se estructura como una nueva oferta de servicios parcialmente intervenida a través de los procesos de regulación, gestión y de demanda desarrollados por las administraciones competentes. A partir de este momento se desarrolla una actividad próspera y en constante crecimiento, pero con importantes problemas de estructuración, que se acentúan con el advenimiento de la actual crisis económica. El establecimiento de pequeñas empresas muy atomizadas, la excesiva temporalidad del empleo, la

ARBOR Vol. 188753 enero-febrero [2012] 135-152 ISSN: 0210-1963 
escasez de convenios profesionales y la excesiva dependencia del sector de la construcción hace que esta actividad se vuelva vulnerable a las coyunturas económicas, como la representada por la actual crisis económica, que supone un freno al proceso de crecimiento y de consolidación de este mercado, salvo para un conjunto de empresas de mayor tamaño (10\%) que se muestran más estables.

De todo ello se deduce que, si bien la creación de la arqueología comercial se produce a través de un importante proceso de innovación institucional que repercute en el entorno social y económico, a través del análisis de la evolución de esta actividad se constata que los factores que están determinando su desarrollo están estrechamente vinculados a la actual coyuntura económica y política, que paraliza la inversión pública y afecta especialmente al sector de la construcción. Por lo tanto, este débil tejido estructural, que funcionaba positivamente en periodos de bonanza económica, en combinación con los efectos de la crisis global, está dificultando el proceso de maduración y de consolidación de este mercado, traduciéndose en elevadas cotas de inestabilidad laboral y económica para este sector.

A pesar de este arduo contexto, cabe resaltar que la potencialidad más importante de esta actividad es su caracterización como servicio intensivo en conocimiento. Es decir, el "producto" con el que trabaja la arqueología comercial es resultado de un conocimiento experto y especializado capaz de adaptarse y reestructurarse en función la demanda. El problema inmediato es que estas empresas no se reconocen como tal por desconocer los procesos, modelos y oportunidades vinculados con este tipo de servicios tan importantes en términos socioeconómicos, sobre todo en el contexto actual basado en la sociedad del conocimiento, en donde la economía de la cultura, las industrias culturales y las industrias patrimoniales se están abriendo hueco y demostrando su importancia a través del impacto que están generando en la economía de los países y en el bienestar social. Por lo tanto, a través de este estudio se constata la importancia del entorno institucional en la creación del entorno industrial, pero también se observa que la evolución de esta actividad está influenciada por las dinámicas del mercado, sobre todo para las empresas de menor tamaño, que en este caso son la gran mayoría. Las empresas que muestran menor vulnerabilidad se caracterizan por disponer de elementos relacionados con la innovación, especialmente por una mayor capacidad de absorción de conocimientos, por una mayor masa crítica y por la adaptación a otras actividades complementarias.

Las implicaciones del estudio apuntan al importante papel que llevan a cabo las instituciones, como agentes que configuran los marcos estructurales en los que los mercados desarrollan su actividad, y la necesidad de este tipo de análisis para comprender los diferentes procesos de producción y de innovación que tienen lugar en los sectores y en los territorios cuando las relaciones de oferta y demanda no se explican por las funciones del mercado. La débil estructuración de este sector desarrollado durante un período de bonanza económica, sin fortalecer mecanismos asociativos, sin convenios ni acuerdos para el desarrollo de la profesión y para la realización efectiva de la actividad productiva, está teniendo efectos negativos para el desarrollo de esta actividad comercial. De aquí se deriva que desde el entorno institucional es importante desarrollar marcos estructurales que favorezcan el desarrollo de la innovación y la competencia empresarial de manera independiente a la actuación pública que los impulsa. En relación al sector arqueológico se observa la inexistencia de un modelo de gestión patrimonial homogéneo y el establecimiento de un complejo marco legal-administrativo.

Del mismo modo, la ausencia de una definición estandarizada de la profesión arqueológica (conocimientos, experiencia y metodología) y de los parámetros de la gestión del patrimonio está dificultando el desarrollo de una oferta de servicios unificada, de mayor tamaño y capaz de competir en el ámbito nacional e internacional. Además, es necesario recordar que el sector público es un importante consumidor de servicios arqueológicos, por lo que el volumen de demanda generado incidirá en el desarrollo de la actividad comercial. Actualmente, la estrecha relación entre el sector arqueológico y el de la construcción (principal usuario de servicios arqueológicos desde el ámbito privado), está generando graves consecuencias para la estabilidad del mercado arqueológico. Para mitigar este efecto, las empresas de arqueología deberian diversificar su oferta de servicios, reorientándose hacia la gestión de recursos culturales que, además de ser una alternativa de negocio, potencia la gestión integral del patrimonio desde la sostenibilidad y tiene posibilidades de generar un importante impacto socioeconómico. 
Por todas estas razones, parece importante el establecimiento de canales de comunicación entre todos los agentes del sector, siendo esencial la imbricación entre el entorno industrial e institucional, pero también el académico, para una mayor potenciación de los recursos.
Igualmente, es prioritario el establecimiento de protocolos de actuación y coordinación interinstitucional para la mejora de las relaciones entre agentes que favorezca vías alternativas para el desarrollo de una gestión integral del patrimonio.

\section{BIBLIOGRAFÍA}

Dosi, G.; Fagiolo, G. y Marengo, L. (1998): "Modelli ed evidenza empirica su quel poco che sappiamo dell'apprendimento in mondi che cambiano", en Vercelli, A. (Ed.), Incertezza, Racionalita e Decisión, II Mulino, Bologna.

Dosi, G.; Orsenigo, L. y Labini, M. (2005): "Technology and the Economy", en Smelser, J. y Swedberg, R., The Handbook of Economy Sociology, Princeton, Princeton University Press, pp. 265271.

Edquist, C. (2005): "Systems of Innovation. Perspectives and Challenges", en Nelson et al., The Oxford Handbook of Innovation, Oxford, Oxford University Press, pp. 181-208.

Eurostat (2007): Cultural Statistics 2007, Luxemburgo, Eurostat Pocketbooks.

Foray, D. (2004): The Economics of Knowledge, Cambridge, MIT Press.

Freeman, C. (1987): Technology, Policy and Economic Performance: lessons from Japan, New York, Frances Printer Publishers.

Frey, L.; Botan, C. y Kreps, G. (2000): Investigating communication: An introduction to research methods, 2. ${ }^{\text {a }}$ ed., Boston, Allyn \& Bacon.

González López, M. (2007): A innovación nos servizos a empresas intensivos en coñecemento e os servizos a empresas intensivos en coñecemento na innovación. A diversidade da innovación: unha perspectiva sectorial, Santiago de Compostela, Universidad de Santiago de Compostela.

Granovetter, M. (1985): "Economic Action and Social Structure: The Problem of Embeddedness", American Journal of Sociology, Vol. 91(3): 481-510.

Greffe, X. (1990): La valeur économique du patrimoine, París, Económica.

Gregersen, B. (1992): "The Public Sector as a Pacer in National Systems of Innovation", en Lundvall, B., National Systems of Innovation: Towards a Theory of Innovation and Interactive Learning, London, Pinter Publishers, pp. 133-150.

Lundvall, B. A. (1992): National Systems of Innovation: Towards a Theory of Innovation and Interactive Learning, London, Pinter Publishers.

Malerba, F. (1992): "Learning by firms and incremental technical change", The Economic Journal, Vol, 102, n. ${ }^{\circ} 413$, pp. 845-859.

Miles, I. et al. (1995): "Knowledge-Intensive Business Services: Users, Carriers and Sources of Innovation", European Innovation Monitoring System. EIMS Publication, n. ${ }^{\circ}$ 15, Luxemburgo.

Metcalfe, J. (1998): "Evolutionary Economics and Creative Destruction", Journal of Bioeconomics, Vol. 3, n. ${ }^{\circ}$, pp. 71-77.

Nelson, R. y Winter, S. (1982): An Evolutionary Theory of Economic Change, Harvard, Belknap Press of Harvard University.

OCDE (2005): Manual de Oslo. Guía para la recogida e interpretación de datos sobre innovación, Paris (traducción
Aceptado: 20 de agosto de 2011 
española-Grupo Tragsa): OCDE http:// www.conacyt.gob.sv/Indicadores $\% 20$ Sector\%20Academcio/Manual_de_ Oslo\%2005.pdf.

Parga-Dans, E. (2011): Innovación y emergencia de un servicio intensivo en conocimiento: El caso de la arqueología comercial, Santiago de Compostela, Universidad de Santiago de Compostela.

Querol, M. y Martínez, B. (1996): La gestión del Patrimonio Arqueológico en España, Madrid, Alianza Editorial.

Querol, M. (2010): Manual de Gestión del Patrimonio Cultural, Madrid, Ediciones Akal.
Smelser, N. y Swedberg, R. (2005): The Handbook of Economic Sociology, Princeton, Princeton Universtity Press.

Teece, D. y Pisano, G. (1994): "The Dynamic Capabilities of Firms: an Introduction. Industrial an Corporate Change", Oxford Journals, Vol. 3, n. 3, pp. 537-556.

Thomi, W. y Böhn, T. (2003): "Knowledge Intensive Business Services in Regional Systems of Innovation-Initial Results from the Case of Southeast-Finland", 43. European Congress of the Regional Science Association, Finland.

Vence Deza, X. y González López, M. (2009): "Concentración Regional de
Ios Servicios a Empresas Intensivos en Conocimiento en España", Papeles de Economía Española, 120: 300318.

Vicente Hernández, E. (2007): Economía del Patrimonio Cultural y Políticas Patrimoniales. Un Estudio de la Política del Patrimonio Arquitectónico en Castilla y León, Madrid, Instituto de Estudios Fiscales.

Windrum, P. y Thomlinson, M. (1998): "The Impact of KIBS on International Competitiveness: A UK-Netherlands Comparison", SI4S Topical Paper, n. ${ }^{0} 10$, STEP Group. 